\title{
Topology of an anti-parity-time symmetric non-Hermitian Su-Schrieffer-Heeger model
}

\author{
H. C. Wu, L. Jin $\odot, *$ and Z. Song $\odot$ \\ School of Physics, Nankai University, Tianjin 300071, China
}

(Received 10 April 2021; revised 17 May 2021; accepted 20 May 2021; published 3 June 2021)

\begin{abstract}
We propose an anti-parity-time (anti- $\mathcal{P} \mathcal{T}$ ) symmetric non-Hermitian Su-Schrieffer-Heeger (SSH) model, where the large non-Hermiticity constructively creates nontrivial topology and greatly expands the topological phase. In the anti- $\mathcal{P} \mathcal{T}$-symmetric SSH model, the gain and loss are alternatively arranged in pairs under the inversion symmetry. The appearance of the degenerate point at the center of the Brillouin zone determines the topological phase transition, whereas the exceptional points unaffect the band topology. The large nonHermiticity leads to unbalanced wave-function distribution in the broken anti- $\mathcal{P} \mathcal{T}$-symmetric phase and induces the nontrivial topology. Our findings can be verified through introducing dissipations in every other two sites of the standard SSH model even in its trivial phase where the nontrivial topology is solely induced by the dissipations.
\end{abstract}

DOI: 10.1103/PhysRevB.103.235110

\section{INTRODUCTION}

The discrete symmetries classify the Hermitian topological phases into 10 folds [1] and classify the non-Hermitian topological phases into 38 folds [2]. The many interesting topological properties of non-Hermitian phases have been reported [3], including the topological phase transitions [4,5], non-Hermitian band theory [6-11], topological insulators [12-19], topological metals [20-22], topological semimetals [23-28], topological invariants [29-37], and topological edge modes [38-46]. The bulk boundary correspondence (BBC) and bulk topological invariant play important roles in the topological characterization. However, in non-Hermitian systems with skin effect [47-61], the spectra between the open-boundary condition $(\mathrm{OBC})$ and the periodic-boundary condition (PBC) can be dramatically distinct, and the conventional $\mathrm{BBC}$ is invalid because of the Aharonov-Bohm effect with imaginary magnetic flux [57]. To correctly describe the spectrum under the $\mathrm{OBC}$, the non-Bloch band theory is developed [58,62-64], the quasimomentum becomes complex and varies on a generalized Brillouin zone (GBZ). A universal analytical method to obtain the GBZ is given for one-dimensional non-Hermitian systems [65]. In the presence of the non-Hermitian skin effect, the damping becomes unidirectional [66]. Zener tunneling becomes chiral at the non-Bloch collapse point [67]. The relation between edge modes and bulk topology is formulated using the Green's-function method [68]. The non-Hermitian topological systems can be implemented in many experimental platforms including the passive (active) photonic crystals of coupled

\footnotetext{
*jinliang@ nankai.edu.cn
}

Published by the American Physical Society under the terms of the Creative Commons Attribution 4.0 International license. Further distribution of this work must maintain attribution to the author(s) and the published article's title, journal citation, and DOI. waveguides [69-75], coupled resonators [76-81], optical lattice [82-84], electronic circuits [85-88], and acoustic lattices $[89,90]$.

The progress on the non-Hermitian Su-Schrieffer-Heeger (SSH) models [88,91-109], Aubry-André-Harper models [110-115], and Rice-Mele (RM) models [116-118] provides fundamental understanding of the non-Hermitian topological phase of matter. In the non-Hermitian SSH model with asymmetric couplings, nonzero imaginary magnetic flux [57], persistent current [63], and non-Hermitian skin effect exist. In the parity-time- $(\mathcal{P} \mathcal{T}$-) symmetric non-Hermitian SSH model with gain and loss [92-98], the $\mathcal{P} \mathcal{T}$ symmetry prevents nonzero imaginary magnetic flux and ensures the BBC. In the exact $\mathcal{P} \mathcal{T}$-symmetric region with a real spectrum, the Berry phase for each separable band is quantized; in the broken $\mathcal{P} \mathcal{T}$-symmetric region with complex spectrum [119], the Berry phase for each separable band is not quantized [97]. Topological interface states are experimentally observed in $\mathcal{P} \mathcal{T}$-symmetric non-Hermitian SSH lattices [120-124].

The anti- $\mathcal{P} \mathcal{T}$ symmetry can also protect the validity of the BBC. In this paper, we propose an anti- $\mathcal{P} \mathcal{T}$-symmetric nonHermitian SSH model through alternatively incorporating the balanced gain and loss under the inversion symmetry in the standard SSH model. The band spectrum becomes partially complex in the presence of non-Hermiticity, indicating the thresholdless anti- $\mathcal{P} \mathcal{T}$-symmetry breaking. The gain and loss help creating the nontrivial topology. The topological characterization and the geometric picture of the topological phases are elaborated. The topological phase transition occurs when the band gap closes and reopens. The degenerated topological edge states have zero energy with a net gain and localized at two lattice boundaries, respectively. Exciting the edge states enable topological lasing [122-128].

\section{MODEL}

The schematic of the non-Hermitian SSH model is shown in Fig. 1(a), which describes a one-dimensional coupled 

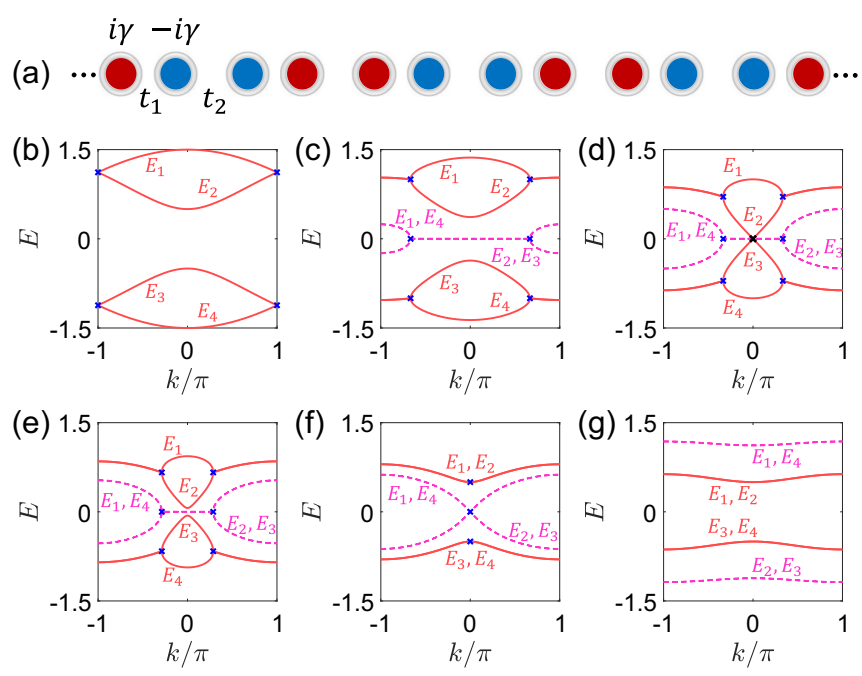

FIG. 1. (a) Schematic of the anti- $\mathcal{P} \mathcal{T}$-symmetric non-Hermitian lattice of a coupled resonator array. The gain (loss) is indicated in red (blue). The spectra are shown in (b) $-(\mathrm{g})$ with system parameters $t_{1}=1, t_{2}=1 / 2$, and $\gamma=0$ for (b), $\gamma=1 / 2$ for (c), $\gamma=\sqrt{3} / 2$ for (d), $\gamma=9 / 10$ for (e), $\gamma=1$ for (f), and $\gamma=3 / 2$ for (g). The red lines indicate the real part of energy, and the magenta dashed lines indicate the imaginary part of energy where the blue crosses indicate the edge degenerate point (DP) for $\gamma=0$ or exceptional points (EPs) for $\gamma \neq 0$, and the black cross indicates a central DP in (d).

resonator array. All the resonators have identical resonant frequency. The staggered distance between the nearestneighbor resonators determines the lattice couplings $t_{1}$ and $t_{2}$ [120-124], which classify two sublattices in the SSH model,

$$
H_{0}=\sum_{j}\left(t_{1} a_{j}^{\dagger} b_{j}+t_{2} b_{j}^{\dagger} a_{j+1}+\text { H.c. }\right)
$$

where $a_{j}^{\dagger}\left(b_{j}^{\dagger}\right)$ and $a_{j}\left(b_{j}\right)$ are the creation and annihilation operators for the sublattice site indexed $j$. To create the anti$\mathcal{P} \mathcal{T}$ symmetry [129-134], the gain and loss are introduced in the resonators under the inversion symmetry in the form of $\{i \gamma,-i \gamma,-i \gamma, i \gamma\}$ in the four-site unit cell,

$$
H_{1}=i \gamma \sum_{j}\left(a_{2 j-1}^{\dagger} a_{2 j-1}-b_{2 j-1}^{\dagger} b_{2 j-1}-a_{2 j}^{\dagger} a_{2 j}+b_{2 j}^{\dagger} b_{2 j}\right) \text {. }
$$

The Hamiltonian of the anti- $\mathcal{P} \mathcal{T}$-symmetric non-Hermitian SSH model reads

$$
H=H_{0}+H_{1} \text {. }
$$

As shown in Fig. 1(a), the anti- $\mathcal{P} \mathcal{T}$-symmetric non-Hermitian $\mathrm{SSH}$ model is invariant under a $\pi$ rotation of the left (right) non-Hermitian dimer and glide half of the unit cell in the translational direction [135].

In comparison to the $\mathcal{P} \mathcal{T}$-symmetric non-Hermitian $\mathrm{SSH}$ model with the gain and loss $\{i \gamma,-i \gamma, i \gamma,-i \gamma\}$ in the four-site unit cell, the only difference between the anti$\mathcal{P} \mathcal{T}$-symmetric and $\mathcal{P} \mathcal{T}$-symmetric SSH models is that the arrangement of the even number of gain and loss pairs; the anti- $\mathcal{P} \mathcal{T}$-symmetric SSH model has the inversion symmetry, whereas the $\mathcal{P} \mathcal{T}$-symmetric SSH model does not.
Interestingly, the role played by the non-Hermiticity $\gamma$ is completely different in these two models. The non-Hermiticity $\gamma$ in the anti- $\mathcal{P} \mathcal{T}$-symmetric SSH model constructively create nontrivial topology. The topologically trivial phase changes into the topologically nontrivial phase as the increasing in non-Hermiticity $\gamma$. The nontrivial topology of the anti- $\mathcal{P} \mathcal{T}$-symmetric SSH model can be directly verified in many experimental platforms that used to demonstrate the $\mathcal{P} \mathcal{T}$-symmetric SSH model. The topological aspect of the anti- $\mathcal{P} \mathcal{T}$-symmetric $\mathrm{SSH}$ model completely differs from that of the nonsymmorphic RM model [136].

Applying the Fourier transformation, the Bloch Hamiltonian of the lattice is obtained as

$$
H_{k}=\left(\begin{array}{cccc}
i \gamma & t_{1} & 0 & t_{2} e^{-i k} \\
t_{1} & -i \gamma & t_{2} & 0 \\
0 & t_{2} & -i \gamma & t_{1} \\
t_{2} e^{i k} & 0 & t_{1} & i \gamma
\end{array}\right)
$$

$H_{k}$ has the anti- $\mathcal{P} \mathcal{T}$-symmetry $(\mathcal{P} \mathcal{T}) H_{k}(\mathcal{P} \mathcal{T})^{-1}=-H_{k}$ with $\mathcal{P}=i \sigma_{x} \otimes \sigma_{y}$, and $\mathcal{T}=\mathcal{K}$ is the complex-conjugation operation. $H_{k}$ also has $\operatorname{TRS}^{\dagger} \mathcal{C}_{+} H_{k}^{\mathrm{T}} \mathcal{C}_{+}^{-1}=H_{-k}$ with $\mathcal{C}_{+}=\sigma_{0} \otimes \sigma_{0}$, PHS $^{\dagger} \mathcal{T}_{-} H_{k}^{*} \mathcal{T}_{-}^{-1}=-H_{-k}$ with $\mathcal{T}_{-}=\sigma_{0} \otimes \sigma_{z}$, and chiral symmetry (pseudo-anti-non-Hermiticity) $\Gamma H_{k}^{\dagger} \Gamma^{-1}=-H_{k}$ with $\Gamma=\mathcal{C}_{+} \mathcal{T}_{-}$, where $\sigma_{0}$ and $\sigma_{x, y, z}$ are the two-by-two identical matrix and Pauli matrix. The system belongs to the $\mathrm{BDI}^{\dagger}$ class in the 38-fold topological classifications of non-Hermitian systems, and the topological phase-transition of the $\mathrm{BDI}^{\dagger}$ class is determined by the closure of the band gap of the real part of energy bands [2]. The interplay between the couplings and the non-Hermiticity alters the band topology and generates the nontrivial topology; furthermore, the loss can solely induce the nontrivial topology if we consider a common gain term $i \gamma$ is removed from $H_{k}$. This greatly simplifies the verification of the anti- $\mathcal{P} \mathcal{T}$-symmetric SSH model in experiments.

In contrast to the $\mathcal{P} \mathcal{T}$ symmetry ensures the energy levels to be conjugate in pairs, the anti- $\mathcal{P} \mathcal{T}$ symmetry ensures the energy levels in pairs with identical imaginary part and opposite real part. The four energy bands are

$$
E_{ \pm, \pm}= \pm \sqrt{t_{1}^{2}+t_{2}^{2}-\gamma^{2} \pm 2 t_{2} \sqrt{t_{1}^{2} \cos ^{2}(k / 2)-\gamma^{2}}}
$$

In the absence of the gain and loss $(\gamma=0)$, the lattice is the Hermitian SSH model. At the topological phase-transition point $t_{1}=t_{2}$, two bands $\pm 2 t_{1} \cos (k / 2)$ of the SSH model are connected at the DP $k= \pm \pi$; the four-band spectrum $E_{ \pm, \pm}$ of $H_{k}$ can be regarded as the spectrum of the SSH model folded at $k= \pm \pi / 2$ and stretched to the entire BZ. Thus, the central band gap closes at the DP at the center of the BZ $k=0$, and the band folding generates another DP at the edge of the BZ $k= \pm \pi$. At $t_{1} \neq t_{2}$, the SSH model is gapped, and the central gap is open as shown in Fig. 1(b); however, the spectrum of $H_{k}$ still has a DP at the edge of BZ protected by the nonsymmorphic symmetry in the four-site unit cell of the SSH model [135].

In the presence of the gain and loss $(\gamma \neq 0)$, the nonHermiticity splits the edge DP into two EPs associated with the anti- $\mathcal{P} \mathcal{T}$-symmetry breaking [Fig. 1(c)]. As the increase in the non-Hermiticity, the two EPs gradually move, and the complex energy region expands from the edge to the center of 

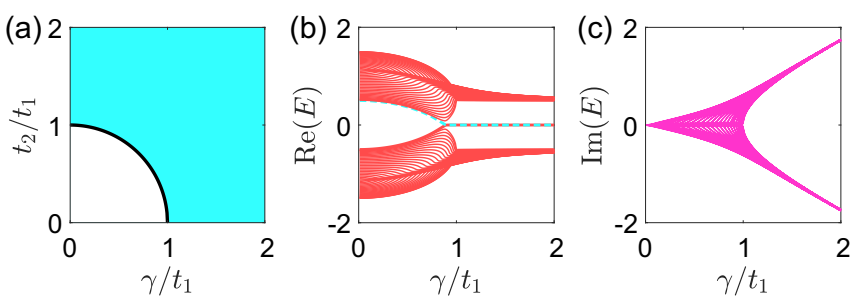

FIG. 2. (a) Phase diagram for the anti- $\mathcal{P} \mathcal{T}$-symmetric nonHermitian SSH model. The real part (b) and the imaginary part (c) of the energy spectrum as a function of the non-Hermiticity; the parameters are $t_{1}=1$ and $t_{2}=1 / 2$.

the BZ as shown in Figs. 1(d) and 1(e). When $\gamma^{2}=t_{1}^{2}$, two EPs merge to one EP at the center of the BZ [Fig. 1(f)] and disappear for $\gamma^{2}>t_{1}^{2}$ [Fig. 1(g)].

The band gap of the central two bands closes at $E=0$ as presented in Fig. 1(d), which requires $\left(\gamma^{2}+t_{2}^{2}-t_{1}^{2}\right)^{2}+$ $4 t_{1}^{2} t_{2}^{2} \sin ^{2}(k / 2)=0$. The central two bands touch at the DP $k=0$ associated with the topological phase transition at the critical non-Hermiticity,

$$
\gamma^{2}+t_{2}^{2}=t_{1}^{2}
$$

\section{PHASE DIAGRAM}

The anti- $\mathcal{P} \mathcal{T}$-symmetric non-Hermitian SSH model reduces to the SSH model for $\gamma=0$, which has the topologically nontrivial phase for $t_{2}^{2}>t_{1}^{2}$ and the topologically trivial phase for $t_{2}^{2}<t_{1}^{2}$. However, the situation changes in the presence of non-Hermiticity as shown in the phase diagram Fig. 2(a). The non-Hermiticity creates the nontrivial topology and the topological region expands in the anti- $\mathcal{P} \mathcal{T}$ symmetric non-Hermitian SSH model, where $\gamma^{2}+t_{2}^{2}<t_{1}^{2}$ is the topologically trivial phase and $\gamma^{2}+t_{2}^{2}>t_{1}^{2}$ is the topologically nontrivial phase. The nontrivial topology of the anti- $\mathcal{P} \mathcal{T}$-symmetric SSH model can be solely created by the non-Hermiticity because large non-Hermiticity induces unbalanced distributions of the wave-function probability. The non-Hermiticity generates nontrivial topology in the uniform chain at $t_{1}^{2}=t_{2}^{2}$ [137] and even in the trivial phase of the Hermitian SSH model at $t_{2}^{2}<t_{1}^{2}$.

The real part and the imaginary part of the energy bands under the $\mathrm{OBC}$ as a function of the non-Hermiticity are shown in Figs. 2(b) and 2(c), respectively. The anti- $\mathcal{P} \mathcal{T}$-symmetric non-Hermitian SSH lattice under the OBC has one pair of edge states in the topologically nontrivial phase. To further elucidate the band structure, the energy bands on the complex energy plane for the non-Hermitian SSH lattice under the OBC are plotted as shown in Figs. 3(a)-3(f); the corresponding PBC spectra are shown in Figs. 1(b) $-1(\mathrm{~g})$. For $\gamma^{2} \leqslant t_{1}^{2}$ and $\gamma^{2}+t_{2}^{2} \neq t_{1}^{2}$, the real part of the energy bands is gapped (the central gap is open). However, the energy bands $E_{1}$ and $E_{2}\left(E_{3}\right.$ and $\left.E_{4}\right)$ are inseparable [6] because of the existence of edge DP or EPs (blue crosses) as shown in Figs. 1(b), 1(c), 1(e), and 1(f); in this sense, the four energy bands can be regarded as two energy bands $E_{r}$ (cyan) and $E_{l}$ (magenta) according to the real part of energy bands as shown in Figs. 3(a), 3(b), $3(\mathrm{~d})$, and 3(e). For $\gamma^{2}+t_{2}^{2}=t_{1}^{2}$ (topological phase transition), the real gap is closed (the central gap is closed) and the two
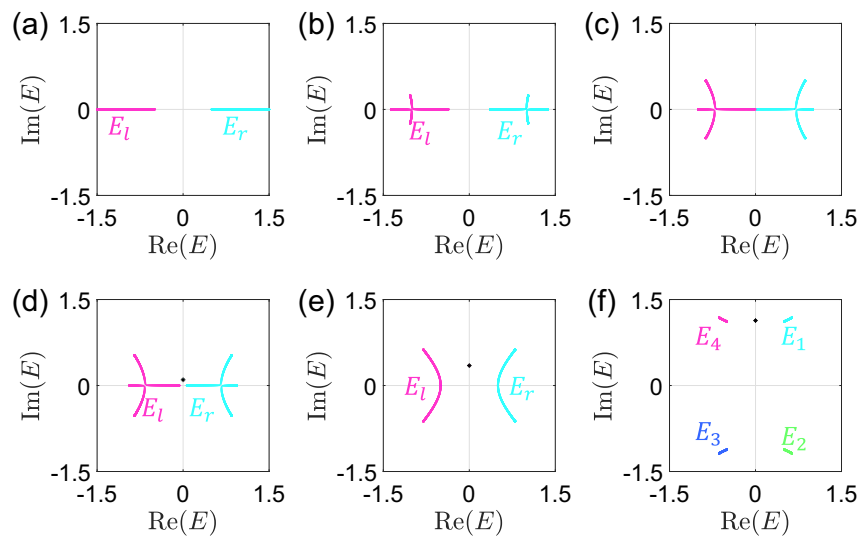

FIG. 3. Energy bands on the complex energy plane under the OBC. The parameters in (a)-(f) are similar as those in Figs. 1(b)$1(\mathrm{~g})$. The black stars indicate a pair of edge states for topological nontrivial phases as shown in (d)-(f).

energy bands $E_{r}$ and $E_{l}$ become single band [Fig. 3(c)]. For $\gamma^{2}>t_{1}^{2}$, the EP disappears between $E_{1}$ and $E_{2}\left(E_{3}\right.$ and $\left.E_{4}\right)$, and the four energy bands are separated [Fig. 3(f)]. In the topologically nontrivial region $\gamma^{2}+t_{2}^{2}>t_{1}^{2}$, there exist one pair of degenerated zero modes (black stars) with gain for the lattice under the OBC as shown in Figs. 3(d)-3(f).

\section{EDGE STATE}

The inversion symmetry and $\mathrm{TRS}^{\dagger}$ ensure the validity of the conventional BBC because both of them lead to real wave numbers for the anti- $\mathcal{P} \mathcal{T}$-symmetric non-Hermitian lattice under the OBC $[2,57]$. The lattice under the OBC supports two degenerate edge states localized at the left and right boundaries of the lattice, respectively. The eigenvalues of edge states are imaginary,

$$
\varepsilon=i\left\{\lambda+\left[4 \gamma^{2}-3\left(t_{2}^{2}+t_{1}^{2}\right)\right] / \lambda-\gamma\right\} / 3,
$$

where $\lambda=\sqrt[3]{\alpha+\sqrt{\beta}}$ with $\alpha=\left(18 t_{2}^{2}+8 \gamma^{2}-9 t_{1}^{2}\right) \gamma$ and $\beta=27\left\{\left[4\left(2 t_{2}^{2}+4 \gamma^{2}-5 t_{1}^{2}\right) t_{2}^{2}-t_{1}^{4}\right] \gamma^{2}+\left(t_{1}^{2}+t_{2}^{2}\right)^{3}\right\}$. The left edge state localizes at the left boundary of the lattice. Without loss of generality, the wave functions in the $j$ th unit cell of the left edge state $\left|\psi_{L}\right\rangle$ can be expressed as $\chi^{j-1}\{1,(\varepsilon-$ $\left.i \gamma) / t_{1},-\chi t_{2} / t_{1}, 0\right\}$ with $\chi=(i \gamma-\varepsilon) /(i \gamma+\varepsilon)$. For $\gamma=0$, the edge states reduce to zero modes with $\varepsilon=0$ and the wave functions in the $j$ th unit cell as $\left(t_{1} / t_{2}\right)^{j-1}\left\{1,0,-t_{1} / t_{2}, 0\right\}$. The right edge state $\left|\psi_{R}\right\rangle$ is the mirror reflection of the left edge state $\left|\psi_{L}\right\rangle$. The edge states have net gain rate and are useful for topological lasing.

\section{GEOMETRIC PICTURE OF BAND TOPOLOGY}

The topology of the anti- $\mathcal{P} \mathcal{T}$-symmetric non-Hermitian SSH model relates to the geometry of the Bloch Hamiltonian winding around the degeneracy points in a two-dimensional parameter space, we show how the non-Hermiticity creates the nontrivial topology. For the Bloch Hamiltonian Eq. (3), we replace $e^{i k}$ by $h_{x}+i h_{y}$ to create a two-dimensional parameter 

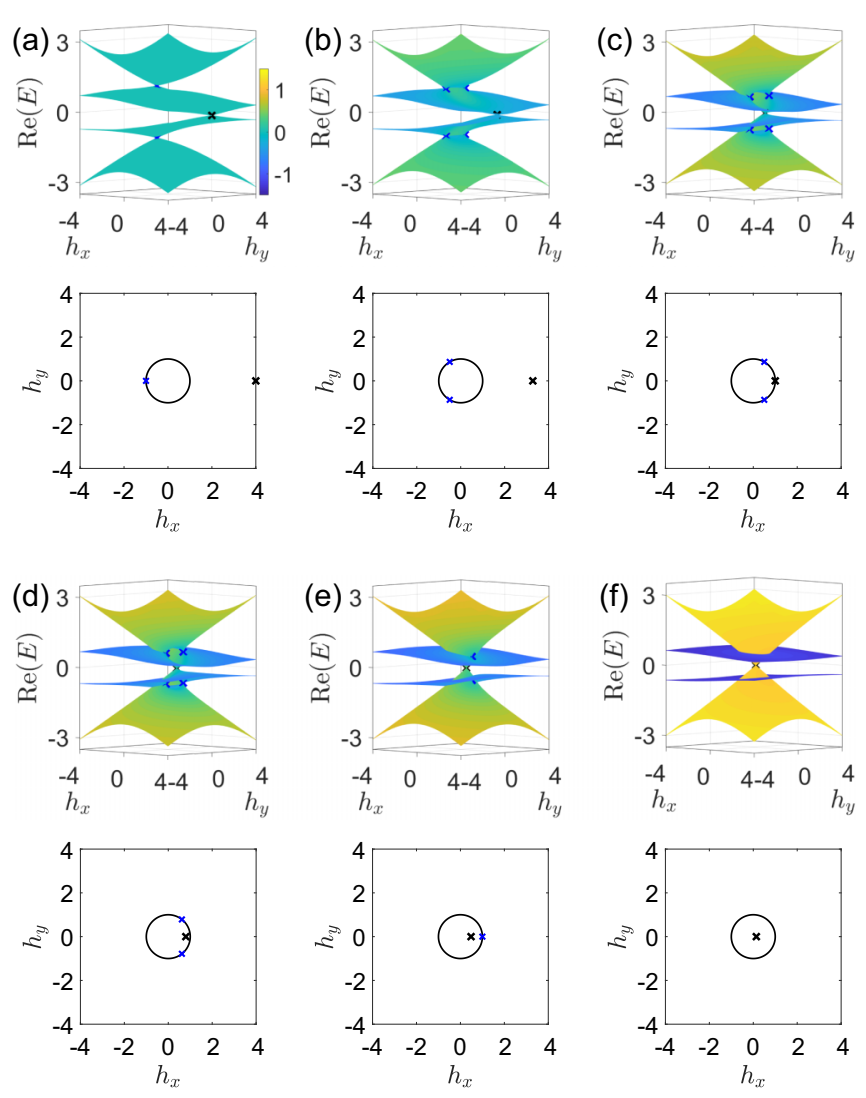

FIG. 4. Complex energy bands and geometric picture of the topology in the two-dimensional parameter space $\left(h_{x}, h_{y}\right)$ for the anti- $\mathcal{P} \mathcal{T}$-symmetric non-Hermitian SSH model. The upper panels depict the complex energy bands where the value indicates the real part and the color indicates the imaginary part of the energy; the lower panels depict the geometric picture of the topology. The unit circle indicates the anti- $\mathcal{P} \mathcal{T}$-symmetric non-Hermitian SSH model, the blue crosses indicate the edge DP or EPs, and the black crosses indicate the central DP. The parameters are (a) $\gamma=0$, (b) $\gamma=1 / 2$, (c) $\gamma=\sqrt{3} / 2$, (d) $\gamma=9 / 10$, (e) $\gamma=1$, and (f) $\gamma=3 / 2$; other parameters are $t_{1}=1$ and $t_{2}=1 / 2$.

space $\left(h_{x}, h_{y}\right)$,

$$
H\left(h_{x}, h_{y}\right)=\left(\begin{array}{cccc}
i \gamma & t_{1} & 0 & t_{2}\left(h_{x}-i h_{y}\right) \\
t_{1} & -i \gamma & t_{2} & 0 \\
0 & t_{2} & -i \gamma & t_{1} \\
t_{2}\left(h_{x}+i h_{y}\right) & 0 & t_{1} & i \gamma
\end{array}\right),
$$

where the $k$-dependent Bloch Hamiltonian Eq. (3) corresponds to a unit circle $h_{x}^{2}+h_{y}^{2}=1$ in the two-dimensional parameter space $\left(h_{x}, h_{y}\right)$.

Figure 4 depicts the complex energy bands extended to the two-dimensional parameter space $\left(h_{x}, h_{y}\right)$ for the anti$\mathcal{P} \mathcal{T}$-symmetric non-Hermitian SSH model at fixed parameters $t_{1}=1$ and $t_{2}=1 / 2$ for the different non-Hermiticity $\gamma$. The edge DP (blue cross) on the unit circle at $\gamma=0$ splits into two EPs on the unit circle at nonzero non-Hermiticity $\gamma<1$; and the two EPs are symmetrically distributed about $h_{y}=0$ because the EPs are symmetrically distributed about $k=0$ in the BZ [see Figs. 1(c)-1(e)]. As the non-Hermiticity increases, the two EPs move on the unit circle from $\left(h_{x}, h_{y}\right)=(-1,0)$ to
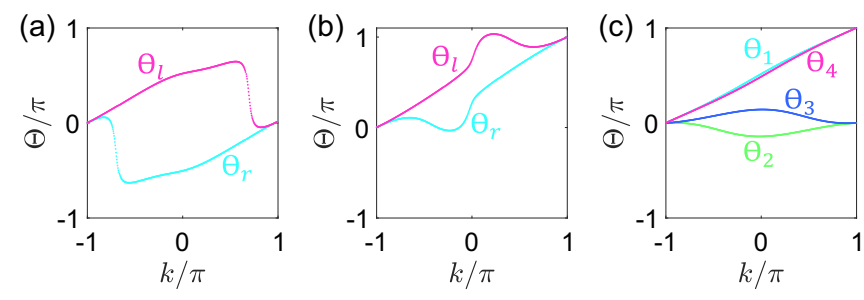

FIG. 5. Zak phase and partial global Zak phase. The parameters are $t_{1}=1, t_{2}=1 / 2$, and $\gamma=1 / 2$ for (a), $\gamma=9 / 10$ for (b), and $\gamma=$ $3 / 2$ for (c).

$\left(h_{x}, h_{y}\right)=(1,0)$; at $\gamma=1$, the two EPs merge into single EP at $\left(h_{x}, h_{y}\right)=(1,0)$; and the EP vanishes for $\gamma>1$. The edge DP or the EPs remain on the unit circle, and the topology is fully determined by the central DP (black cross). From the unit circle winding around the central DP, we can observe how the nontrivial topology is created by the non-Hermiticity $\gamma$. For $\gamma=0$ in Fig. 4(a), the central DP is outside the unit circle; thus, the system is in the topologically trivial phase. The nonzero non-Hermiticity $\gamma$ moves the central DP along $h_{y}=0$ towards the negative $h_{x}$ direction in the parameter space. For $\gamma=1 / 2$ in Fig. 4(b), the central DP moves to $\left(h_{x}, h_{y}\right)=(3.284,0)$, and the system enters the white region of the phase diagram as shown in Fig. 2(a). For $\gamma=\sqrt{3} / 2$ in Fig. 4(c), the central DP moves to $\left(h_{x}, h_{y}\right)=(1,0)$ and locates on the unit circle; the system is at the boundary of the white and cyan regions. For $\gamma=9 / 10$ in Fig. 4(d), the central DP is enclosed in the unit circle, the topology of the system changes, and the system enters the cyan region. For $\gamma=1$ in Fig. 4(e), the central DP keeps inside the unit circle; the system is in the nontrivial phase for the nonzero $t_{2}$, but the energy bands are not completely separated. For $\gamma=3 / 2$ in Fig. 4(f), the central DP is still inside the unit circle; in this situation, the four bands are completely separated and the system is in the nontrivial phase.

\section{ZAK PHASE AND PARTIAL GLOBAL ZAK PHASE}

When the four complex energy bands are separated at $\gamma^{2}>$ $t_{1}^{2}$, each energy band is associated with a Zak phase,

$$
\Theta_{n}=i \oint d k\left\langle\varphi_{n}\left|\partial_{k}\right| \psi_{n}\right\rangle
$$

In the definition of $\Theta_{n},\left|\varphi_{n}\right\rangle$ is the left eigenstate, and $\left|\psi_{n}\right\rangle$ is the right eigenstate, $H_{k}\left|\psi_{n}\right\rangle=E_{n}\left|\psi_{n}\right\rangle$ and $H_{k}^{\dagger}\left|\varphi_{n}\right\rangle=E_{n}^{*}\left|\varphi_{n}\right\rangle$, where the subscript $n$ is the band index. $E_{1}$ denotes the band with positive real and imaginary energies, $E_{2}$ denotes the band with positive real and negative imaginary energies, $E_{3}$ denotes the band with negative real and imaginary energies, and $E_{4}$ denotes the band with negative real and positive imaginary energies as shown in Figs. 3(f) and Fig. 1(g). Their wave functions are $\left|\psi_{1}\right\rangle,\left|\psi_{2}\right\rangle,\left|\psi_{3}\right\rangle$, and $\left|\psi_{4}\right\rangle$, respectively. The system has the inversion symmetry, which ensures that the Zak phase for each separated energy band is an integer of $\pi$. Thus, the Zak phase is used for topological characterization. In Fig. 5(c), the Zak phases for the bands $E_{1}$ and $E_{4}$ are $\pi$, and the Zak phases for the bands $E_{2}$ and $E_{3}$ are 0 ; which 
are consistent with the geometric picture in Fig. 4(f), the central DP belongs to energy bands $E_{1}$ and $E_{4}$ and predicts the existence of one pair of the topological zero modes with gain for the system under the OBC.

For the energy bands embedded with EPs $\left(\gamma^{2} \leqslant t_{1}^{2}\right.$ and $\gamma^{2}+t_{2}^{2} \neq t_{1}^{2}$ ), there are only two energy bands $E_{r}$ and $E_{l}$. The two-state coalescence EP $2_{12}$ exists only in the energy bands $E_{1}$ and $E_{2}$, and the two-state coalescence EP2 ${ }_{34}$ exists only in the energy bands $E_{3}$ and $E_{4}$. In this sense, we define two partial global Zak phases,

$$
\Theta_{r}=\Theta_{1}+\Theta_{2} ; \Theta_{l}=\Theta_{3}+\Theta_{4} .
$$

In the calculation of the partial global Zak phase, the momentum ranges $\left[k_{\mathrm{EP}}-\Delta k, k_{\mathrm{EP}}+\Delta k\right]$ are removed because that the coalesced wave functions are self-orthogonal at the EPs [137], where $\Delta k$ is an infinite small positive real number. The partial global Zak phase is valid for the topological characterization.

For $\gamma^{2} \leqslant t_{1}^{2}$ and $\gamma^{2}+t_{2}^{2}<t_{1}^{2}$, both partial global Zak phases $\Theta_{r}$ and $\Theta_{l}$ are 0 as shown in Fig. 5(a). This indicates the phase is topologically trivial without any edge state under the OBC. However, for $\gamma^{2} \leqslant t_{1}^{2}$ and $\gamma^{2}+t_{2}^{2}>t_{1}^{2}$, both partial global Zak phases $\Theta_{r}$ and $\Theta_{l}$ are $\pi$ as shown in Fig. 5(b). This indicates the phase is topologically nontrivial and one pair of topological edge states appear under the OBC.

\section{CONCLUSION}

We propose the anti- $\mathcal{P} \mathcal{T}$-symmetric non-Hermitian SSH model as a prototypical anti- $\mathcal{P} \mathcal{T}$-symmetric topological lattice. The gain and loss are alternatively introduced in pairs in the standard SSH model through holding the inversion symmetry. The inversion symmetric gain and loss result in the thresholdless breaking of anti- $\mathcal{P} \mathcal{T}$ symmetry, and the energy spectrum is partially or fully complex. We provide novel insights on the roles played by the anti- $\mathcal{P} \mathcal{T}$ symmetry and non-Hermiticity in the topological phases. The large non-Hermiticity constructively creates the nontrivial topology and greatly expands the topologically nontrivial region of the SSH model. The topological edge states localized at two boundaries of the lattice are degenerate and suitable for topological lasing. Besides, the dissipation can solely induce the nontrivial topology. In comparison to the $\mathcal{P} \mathcal{T}$-symmetric non-Hermitian SSH model, only the arrangement of gain and loss in the anti- $\mathcal{P} \mathcal{T}$-symmetric non-Hermitian SSH model is different; the proposed anti- $\mathcal{P} \mathcal{T}$-symmetric non-Hermitian SSH model can be easily implemented in the microring resonator arrays, coupled optical waveguides, photonic crystals, electronic circuits, and acoustic lattices [69-87,89,90].

\section{ACKNOWLEDGMENTS}

This work was supported by the National Natural Science Foundation of China (Grants No. 11975128 and No. 11874225).
[1] C.-K. Chiu, J. C. Y. Teo, A. P. Schnyder, and S. Ryu, Classification of topological quantum matter with symmetries, Rev. Mod. Phys. 88, 035005 (2016).

[2] K. Kawabata, K. Shiozaki, M. Ueda, and M. Sato, Symmetry and Topology in Non-Hermitian Physics, Phys. Rev. X 9, 041015 (2019).

[3] Y. Ashida, Z. Gong, M. Ueda, Non-Hermitian Physics, Adv. Phys. 69, 249 (2020).

[4] M. S. Rudner and L. S. Levitov, Topological Transition in a Non-Hermitian Quantum Walk, Phys. Rev. Lett. 102, 065703 (2009).

[5] J. Ren and N. A. Sinitsyn, Braid group and topological phase transitions in nonequilibrium stochastic dynamics, Phys. Rev. E 87, 050101(R) (2013).

[6] H. Shen, B. Zhen, and L. Fu, Topological Band Theory for Non-Hermitian Hamiltonians, Phys. Rev. Lett. 120, 146402 (2018).

[7] K. Yamamoto, M. Nakagawa, K. Adachi, K. Takasan, M. Ueda, and N. Kawakami, Theory of Non-Hermitian Fermionic Superfluidity with a Complex-Valued Interaction, Phys. Rev. Lett. 123, 123601 (2019).

[8] Z.-Y. Ge, Y.-R. Zhang, T. Liu, S.-W. Li, H. Fan, and F. Nori, Topological band theory for non-Hermitian systems from the Dirac equation, Phys. Rev. B 100, 054105 (2019).

[9] C. C. Wojcik, X.-Q. Sun, T. Bzdušek, and S. Fan, Homotopy characterization of non-Hermitian Hamiltonians, Phys. Rev. B 101, 205417 (2020).
[10] C.-X. Guo, X.-R. Wang, C. Wang, and S.-P. Kou, NonHermitian dynamic strings and anomalous topological degeneracy on a non-Hermitian toric-code model with parity-time symmetry, Phys. Rev. B 101, 144439 (2020).

[11] C.-X. Guo, X.-R. Wang, and S.-P. Kou, Non-Hermitian avalanche effect: Non-perturbative effect induced by local non-Hermitian perturbation on a Z2 topological order, Europhys. Lett. 131, 27002 (2020).

[12] D.-W. Zhang, L.-Z. Tang, L.-J. Lang, H. Yan, and S.-L. Zhu, Non-Hermitian topological Anderson insulators, Sci. China: Phys., Mech. Astron. 63, 267062 (2020).

[13] T. M. Philip, M. R. Hirsbrunner, and M. J. Gilbert, Loss of hall conductivity quantization in a non-Hermitian quantum anomalous hall insulator, Phys. Rev. B 98, 155430 (2018).

[14] Y. Chen and H. Zhai, Hall conductance of a non-Hermitian Chern insulator, Phys. Rev. B 98, 245130 (2018).

[15] M. R. Hirsbrunner, T. M. Philip, and M. J. Gilbert, Topology and observables of the non-Hermitian Chern insulator, Phys. Rev. B 100, 081104(R) (2019).

[16] E. J. Bergholtz and J. C. Budich, Non-Hermitian Weyl physics in topological insulator ferromagnet junctions, Phys. Rev. Research 1, 012003(R) (2019).

[17] K. Kawabata, Y. Ashida, H. Katsura, and M. Ueda, Paritytime-symmetric topological superconductor, Phys. Rev. B 98, 085116 (2018).

[18] Z. Xu and S. Chen, Topological Bose-Mott insulators in onedimensional non-Hermitian superlattices, Phys. Rev. B 102, 035153 (2020). 
[19] J. Hou, Y.-J. Wu, and C. Zhang, Two-dimensional nonHermitian topological phases induced by asymmetric hopping in a one-dimensional superlattice, Phys. Rev. A 103, 033305 (2021).

[20] X. Zhang, G. Li, Y. Liu, T. Tai, R. Thomale, and C. H. Lee, Tidal surface states as fingerprints of non-Hermitian nodal knot metals, Commun. Phys. 4, 47 (2021).

[21] J. Carlström, M. Stålhammar, J. C. Budich, and E. J. Bergholtz, Knotted non-Hermitian metals, Phys. Rev. B 99, 161115(R) (2019).

[22] K. Wang, L. Xiao, J. C. Budich, W. Yi, P. Xue, Simulating Exceptional Non-Hermitian Metals with Single-Photon Interferometry, arXiv:2011.01884.

[23] Y. Xu, S.-T. Wang, and L.-M. Duan, Weyl Exceptional Rings in a Three-Dimensional Dissipative Cold Atomic Gas, Phys. Rev. Lett. 118, 045701 (2017).

[24] A. Cerjan, M. Xiao, L. Yuan, and S. Fan, Effects of nonHermitian perturbations on Weyl Hamiltonians with arbitrary topological charges, Phys. Rev. B 97, 075128 (2018).

[25] H. Wang, J. Ruan, and H. Zhang, Non-Hermitian nodalline semimetals with an anomalous bulk-boundary correspondence, Phys. Rev. B 99, 075130 (2019).

[26] Z. Yang and J. Hu, Non-Hermitian Hopf-link exceptional line semimetals, Phys. Rev. B 99, 081102(R) (2019).

[27] T. Yoshida, R. Peters, N. Kawakami, and Y. Hatsugai, Symmetry-protected exceptional rings in two-dimensional correlated systems with chiral symmetry, Phys. Rev. B 99, 121101(R) (2019).

[28] K. Yokomizo and S. Murakami, Topological semimetal phase with exceptional points in one-dimensional non-Hermitian systems, Phys. Rev. Research 2, 043045 (2020).

[29] F. Song, S. Yao, and Z. Wang, Non-Hermitian Topological Invariants in Real Space, Phys. Rev. Lett. 123, 246801 (2019).

[30] L.-F. Zhang, L.-Z. Tang, Z.-H. Huang, G.-Q. Zhang, W. Huang, and D.-W. Zhang, Machine learning topological invariants of non-Hermitian systems, Phys. Rev. A 103, 012419 (2021).

[31] D. Leykam, K. Y. Bliokh, C. Huang, Y. D. Chong, and F. Nori, Edge Modes, Degeneracies, and Topological Numbers in NonHermitian Systems, Phys. Rev. Lett. 118, 040401 (2017).

[32] T.-S. Deng and W. Yi, Non-bloch topological invariants in a non-Hermitian domain wall system, Phys. Rev. B 100, 035102 (2019).

[33] X. Z. Zhang and Z. Song, Partial topological Zak phase and dynamical confinement in a non-Hermitian bipartite system, Phys. Rev. A 99, 012113 (2019).

[34] W. Brzezicki and T. Hyart, Hidden Chern number in onedimensional non-Hermitian chiral-symmetric systems, Phys. Rev. B 100, 161105(R) (2019).

[35] J. Claes and T. L. Hughes, Skin effect and winding number in disordered non-Hermitian systems, Phys. Rev. B 103, L140201 (2021).

[36] B. Zhu, Y. Ke, H. Zhong, and C. Lee, Dynamic winding number for exploring band topology, Phys. Rev. Research 2, 023043 (2020).

[37] L. Wang, Q. Liu, and Y. Zhang, Quantum dynamics on a lossy non-Hermitian lattice, Chin. Phys. B 30, 020506 (2021).

[38] K. Esaki, M. Sato, K. Hasebe, and M. Kohmoto, Edge states and topological phases in non-Hermitian systems, Phys. Rev. B 84, 205128 (2011).
[39] C.-E. Bardyn, M. A. Baranov, E. Rico, A. Imamoglu, P. Zoller, and S. Diehl, Majorana Modes in Driven-Dissipative Atomic Superfluids with a Zero Chern Number, Phys. Rev. Lett. 109, 130402 (2012).

[40] Y. V. Kartashov, V. V. Konotop, and L. Torner, Topological States in Partially- $\mathcal{P} \mathcal{T}$-Symmetric Azimuthal Potentials, Phys. Rev. Lett. 115, 193902 (2015).

[41] S. Malzard, C. Poli, and H. Schomerus, Topologically Protected Defect States in Open Photonic Systems with NonHermitian Charge-Conjugation and Parity-Time-Symmetry, Phys. Rev. Lett. 115, 200402 (2015).

[42] L. Xiao, X. Zhan, Z. H. Bian, K. K. Wang, X. Zhang, X. P. Wang, J. Li, K. Mochizuki, D. Kim, N. Kawakami, W. Yi, H. Obuse, B. C. Sanders, and P. Xue, Observation of topological edge states in parity-time-symmetric quantum walks, Nat. Phys. 13, 1117 (2017).

[43] X.-R. Wang, C.-X. Guo, and S.-P. Kou, Defective edge states and number-anomalous bulk-boundary correspondence in non-Hermitian topological systems, Phys. Rev. B 101, 121116(R) (2020).

[44] K. Sone, Y. Ashida, and T. Sagawa, Exceptional nonHermitian topological edge mode and its application to active matter, Nat. Commun. 11, 5745 (2020).

[45] S. Lieu, Non-Hermitian Majorana modes protect degenerate steady states, Phys. Rev. B 100, 085110 (2019).

[46] B. Wang, T. Chen, and X. Zhang, Observation of Novel Robust Edge States in Dissipative Non-Hermitian Quantum Walks, Laser Photonics Rev. 14, 2000092 (2020).

[47] F. K. Kunst, E. Edvardsson, J. C. Budich, and E. J. Bergholtz, Biorthogonal Bulk-Boundary Correspondence in Non-Hermitian Systems, Phys. Rev. Lett. 121, 026808 (2018).

[48] Z. Gong, Y. Ashida, K. Kawabata, K. Takasan, S. Higashikawa, and M. Ueda, Topological Phases of NonHermitian Systems, Phys. Rev. X 8, 031079 (2018).

[49] N. Okuma, K. Kawabata, K. Shiozaki, and M. Sato, Topological Origin of Non-Hermitian Skin Effects, Phys. Rev. Lett. 124, 086801 (2020).

[50] S. Weidemann, M. Kremer, T. Helbig, T. Hofmann, A. Stegmaier, M. Greiter, R. Thomale, A. Szameit, Topological funneling of light, Science 368, 311 (2020).

[51] L. Xiao, T. S. Deng, K. K. Wang, G. Y. Zhu, Z. Wang, W. Yi, and P. Xue, Observation of non-Hermitian bulk-boundary correspondence in quantum dynamics, Nat. Phys. 16, 761 (2020).

[52] C.-H. Liu, K. Zhang, Z. Yang, and S. Chen, Helical damping and dynamical critical skin effect in open quantum systems, Phys. Rev. Research 2, 043167 (2020).

[53] X. Zhang and J. Gong, Non-Hermitian Floquet topological phases: Exceptional points, coalescent edge modes, and the skin effect, Phys. Rev. B 101, 045415 (2020).

[54] H. Jiang, L.-J. Lang, C. Yang, S.-L. Zhu, and S. Chen, Interplay of non-Hermitian skin effects and Anderson localization in nonreciprocal quasiperiodic lattices, Phys. Rev. B 100, 054301 (2019).

[55] K. Kawabata, M. Sato, and K. Shiozaki, Higher-order nonHermitian skin effect, Phys. Rev. B 102, 205118 (2020).

[56] S. Longhi, Probing non-Hermitian skin effect and non-Bloch phase transitions, Phys. Rev. Research 1, 023013 (2019).

[57] L. Jin and Z. Song, Bulk-boundary correspondence in a nonHermitian system in one dimension with chiral inversion symmetry, Phys. Rev. B 99, 081103(R) (2019). 
[58] S. Yao and Z. Wang, Edge States and Topological Invariants of Non-Hermitian Systems, Phys. Rev. Lett. 121, 086803 (2018).

[59] R. Koch and J. C. Budich, Bulk-boundary correspondence in non-Hermitian systems: stability analysis for generalized boundary conditions, Eur. Phys. J. D 74, 70 (2020).

[60] T. Yoshida, T. Mizoguchi, and Y. Hatsugai, Mirror skin effect and its electric circuit simulation, Phys. Rev. Research 2, 022062(R) (2020).

[61] F. Roccati, Non-Hermitian skin effect as an impurity problem, arXiv:2105.01197.

[62] K. Yokomizo and S. Murakami, Non-Bloch Band Theory of Non-Hermitian Systems, Phys. Rev. Lett. 123, 066404 (2019).

[63] K. Zhang, Z. Yang, and C. Fang, Correspondence between Winding Numbers and Skin Modes in Non-Hermitian Systems, Phys. Rev. Lett. 125, 126402 (2020).

[64] K. Kawabata, N. Okuma, and M. Sato, Non-Bloch band theory of non-Hermitian Hamiltonians in the symplectic class, Phys. Rev. B 101, 195147 (2020).

[65] Z. Yang, K. Zhang, C. Fang, and J. Hu, Non-Hermitian Bulk-Boundary Correspondence and Auxiliary Generalized Brillouin Zone Theory, Phys. Rev. Lett. 125, 226402 (2020).

[66] F. Song, S. Yao, and Z. Wang, Non-Hermitian Skin Effect and Chiral Damping in Open Quantum Systems, Phys. Rev. Lett. 123, 170401 (2019).

[67] S. Longhi, Non-Bloch-Band Collapse and Chiral Zener Tunneling, Phys. Rev. Lett. 124, 066602 (2020).

[68] D. S. Borgnia, A. J. Kruchkov, and R.-J. Slager, NonHermitian Boundary Modes and Topology, Phys. Rev. Lett. 124, 056802 (2020).

[69] A. Szameit, M. C. Rechtsman, O. Bahat-Treidel, and M. Segev, $\mathcal{P} \mathcal{T}$-symmetry in honeycomb photonic lattices, Phys. Rev. A 84, 021806(R) (2011).

[70] S. K. Gupta, Y. Zou, X.-Y. Zhu, M.-H. Lu, L.-J. Zhang, X.-P. Liu, and Y.-F. Chen, Parity-Time Symmetry in Non-Hermitian Complex Optical Media, Adv. Mater. 32, 1903639 (2020).

[71] X. Zhu, H. Wang, S. K. Gupta, H. Zhang, B. Xie, M. Lu, and Y. Chen, Photonic non-Hermitian skin effect and nonBloch bulk-boundary correspondence, Phys. Rev. Research 2, 013280 (2020).

[72] Y.-J. Wu, C.-C. Liu, and J. Hou, Wannier-type photonic higher-order topological corner states induced solely by gain and loss, Phys. Rev. A 101, 043833 (2020).

[73] A. Cerjan, S. Huang, M. Wang, K. P. Chen, Y. Chong, and M. C. Rechtsman, Experimental realization of a Weyl exceptional ring, Nat. Photonics 13, 623 (2019).

[74] B. Zhu, H. Zhong, J. Jia, F. Ye, and L. Fu, Floquet control of global PT symmetry in quadrimer waveguide arrays, Phys. Rev. A 102, 053510 (2020).

[75] C. Qin, Bi. Wang, Z. J. Wong, S. Longhi, and P. Lu, Discrete diffraction and Bloch oscillations in non-Hermitian frequency lattices induced by complex photonic gauge fields, Phys. Rev. B 101, 064303 (2020).

[76] M. Pan, H. Zhao, P. Miao, S. Longhi, and L. Feng, Photonic zero mode in a non-Hermitian photonic lattice, Nat. Commun. 9, 1308 (2018).

[77] J. M. Zeuner, M. C. Rechtsman, Y. Plotnik, Y. Lumer, S. Nolte, M. S. Rudner, M. Segev, and A. Szameit, Observation of a Topological Transition in the Bulk of a Non-Hermitian System, Phys. Rev. Lett. 115, 040402 (2015).
[78] M.-A. Miri and A. Alù, Exceptional points in optics and photonics, Science 363, eaar7709 (2019).

[79] S. Malzard and H. Schomerus, Bulk and edge-state arcs in non-Hermitian coupled-resonator arrays, Phys. Rev. A 98, 033807 (2018).

[80] X. Yin and C. Peng, Manipulating light radiation from a topological perspective, Photonics Res. 8, 11 (2020).

[81] X.-W. Luo and C. Zhang, Higher-Order Topological Corner States Induced by Gain and Loss, Phys. Rev. Lett. 123, 073601 (2019).

[82] L. Li, C. H. Lee, and J. Gong, Topological Switch for NonHermitian Skin Effect in Cold-Atom Systems with Loss, Phys. Rev. Lett. 124, 250402 (2020).

[83] B.-Y. Sun and Z.-W. Zhou, One-dimensional one-band topologically nontrivial non-Hermitian system simulated in optical cavities, Phys. Rev. A 102, 023501 (2020).

[84] E. J. Bergholtz, J. C. Budich, and F. K. Kunst, Exceptional topology of non-Hermitian systems, Rev. Mod. Phys. 93, 015005 (2021).

[85] M. Ezawa, Higher-order topological electric circuits and topological corner resonance on the breathing kagome and pyrochlore lattices, Phys. Rev. B 98, 201402(R) (2018); Braiding of Majorana-like corner states in electric circuits and its non-Hermitian generalization, 100, 045407 (2019).

[86] C. H. Lee, S. Imhof, C. Berger, F. Bayer, J. Brehm, L. W. Molenkamp, T. Kiessling, and R. Thomale, Topolectrical circuits, Commun. Phys. 1, 39 (2018).

[87] X. X. Zhang and M. Franz, Non-Hermitian Exceptional Landau Quantization in Electric Circuits, Phys. Rev. Lett. 124, 046401 (2020).

[88] A. Stegmaier, S. Imhof, T. Helbig, T. Hofmann, C. H. Lee, M. Kremer, A. Fritzsche, T. Feichtner, S. Klembt, S. Höfling, I. Boettcher, I. C. Fulga, O. G. Schmidt, M. Greiter, T. Kiessling, A. Szameit, and R. Thomale, Topological defect engineering and PT-symmetry in non-Hermitian electrical circuits, Phys. Rev. Lett. 126, 215302 (2021).

[89] W. Zhu, X. Fang, D. Li, Y. Sun, Y. Li, Y. Jing, and H. Chen, Simultaneous Observation of a Topological Edge State and Exceptional Point in an Open and Non-Hermitian Acoustic System, Phys. Rev. Lett. 121, 124501 (2018).

[90] H. Gao, H. Xue, Q. Wang, Z. Gu, T. Liu, J. Zhu, and B. Zhang, Observation of topological edge states induced solely by non-Hermiticity in an acoustic crystal, Phys. Rev. B 101, 180303(R) (2020).

[91] S. Lieu, Topological phases in the non-Hermitian Su-Schrieffer-Heeger model, Phys. Rev. B 97, 045106 (2018).

[92] Y. C. Hu and T. L. Hughes, Absence of topological insulator phases in non-Hermitian $\mathcal{P} \mathcal{T}$-symmetric Hamiltonians, Phys. Rev. B 84, 153101 (2011).

[93] H. Schomerus, Topologically protected midgap states in complex photonic lattices, Opt. Lett. 38, 1912 (2013).

[94] B. Zhu, R. Lü, and S. Chen, $\mathcal{P} \mathcal{T}$ symmetry in the nonHermitian Su-Schrieffer-Heeger model with complex boundary potentials, Phys. Rev. A 89, 062102 (2014).

[95] C. Yuce, Topological phase in a non-Hermitian $\mathcal{P} \mathcal{T}$ symmetric system, Phys. Lett. A 379, 1213 (2015).

[96] H. Menke and M. M. Hirschmann, Topological quantum wires with balanced gain and loss, Phys. Rev. B 95, 174506 (2017). 
[97] X. M. Yang, H. C. Wu, P. Wang, L. Jin, and Z. Song, Visualizing one-dimensional non-hermitian topological phases, J. Phys. Commun. 4, 095005 (2020).

[98] Z. Xu, R. Zhang, S. Chen, L. Fu, and Y. Zhang, Fate of zero modes in a finite Su-Schrieffer-Heeger model with $\mathcal{P} \mathcal{T}$ symmetry, Phys. Rev. A 101, 013635 (2020).

[99] L.-J. Lang, Y. Weng, Y. Zhang, E. Cheng, and Q. Liang, Dynamical robustness of topological end states in nonreciprocal Su-Schrieffer-Heeger models with open boundary conditions, Phys. Rev. B 103, 014302 (2021).

[100] K. Xu, X. Zhang, K. Luo, R. Yu, D. Li, and H. Zhang, Coexistence of topological edge states and skin effects in the non-Hermitian Su-Schrieffer-Heeger model with long-range nonreciprocal hopping in topoelectric realizations, Phys. Rev. B 103, 125411 (2021).

[101] S. Li, M. Liu, F. Li, and B. Liu, Topological phase transition of the extended non-Hermitian Su-Schrieffer-Heeger model, Phys. Scr. 96, 015402 (2020).

[102] X.-W. Xu, Y.-Z. Li, Z.-F. Liu, and A.-X. Chen, General bounded corner states in the two-dimensional Su-SchriefferHeeger model with intracellular next-nearest-neighbor hopping, Phys. Rev. A 101, 063839 (2020).

[103] W.-X. Cui, L. Qi, Y. Xing, S. Liu, S. Zhang, and H.-F. Wang, Localized photonic states and dynamic process in nonreciprocal coupled Su-Schrieffer-Heeger chain, Opt. Express 28, 37026 (2020).

[104] L. Du, J.-H. Wu, M. Artoni, and G. C. L. Rocca, Phasedependent topological interface state and spatial adiabatic passage in a generalized Su-Schrieffer-Heeger model, Phys. Rev. A 100, 012112 (2019).

[105] C. Yuce and H. Ramezani, Topological states in a non-Hermitian two-dimensional Su-Schrieffer-Heeger model, Phys. Rev. A 100, 032102 (2019).

[106] H. Wu and J.-H. An, Floquet topological phases of non-Hermitian systems, Phys. Rev. B 102, 041119(R) (2020).

[107] K.-I. Imura and Y. Takane, Generalized bulk-edge correspondence for non-Hermitian topological systems, Phys. Rev. B 100, 165430 (2019).

[108] S. R. Pocock, P. A. Huidobro, and V. Giannini, Bulk-edge correspondence and long-range hopping in the topological plasmonic chain, Nanophotonics 8, 1337 (2019).

[109] H. Jiang, R. Lü and S. Chen, Topological invariants, zero mode edge states and finite size effect for a generalized nonreciprocal Su-Schrieffer-Heeger model, Eur. Phys. J. B 93, 125 (2020).

[110] S. Longhi, Phase transitions in a non-Hermitian Aubry-André Harper model, Phys. Rev. B 103, 054203 (2021).

[111] S. Longhi, Metal-insulator phase transition in a non-Hermitian Aubry-André Harper model, Phys. Rev. B 100, 125157 (2019).

[112] S. Schiffer, X.-J. Liu, H. Hu, and J. Wang, Anderson localization transition in a robust $\mathcal{P} \mathcal{T}$-symmetric phase of a generalized Aubry-André model, Phys. Rev. A 103, L011302 (2021)

[113] Q.-B. Zeng, Y.-B. Yang, and Y. Xu, Topological phases in nonHermitian Aubry-André Harper models, Phys. Rev. B 101, 020201(R) (2020).

[114] D.-W. Zhang, Y.-L. Chen, G.-Q. Zhang, L.-J. Lang, Z. Li, and S.-L. Zhu, Skin superfluid, topological Mott insulators, and asymmetric dynamics in an interacting non-Hermitian AubryAndré Harper model, Phys. Rev. B 101, 235150 (2020).

[115] Q.-B. Zeng and Y. Xu, Winding numbers and generalized mobility edges in non-Hermitian systems, Phys. Rev. Research 2, 033052 (2020).

[116] R. Wang, X. Z. Zhang, and Z. Song, Dynamical topological invariant for the non-Hermitian Rice-Mele model, Phys. Rev. A 98, 042120 (2018).

[117] C. Yuce, Spontaneous topological pumping in non-Hermitian systems, Phys. Rev. A 99, 032109 (2019).

[118] Y. Yi and Z. Yang, Non-Hermitian Skin Modes Induced by On-Site Dissipations and Chiral Tunneling Effect, Phys. Rev. Lett. 125, 186802 (2020).

[119] M. Parto, Y. G. N. Liu, B. Bahari, M. Khajavikhan, and D. N. Christodoulides, Non-Hermitian and topological photonics: optics at an exceptional point, Nanophotonics 10, 403 (2020).

[120] S. Weimann, M. Kremer, Y. Plotnik, Y. Lumer, S. Nolte, K. G. Makris, M. Segev, M. C. Rechtsman, and A. Szameit, Topologically protected bound states in photonic parity-timesymmetric crystals, Nature Mater. 16, 433 (2017).

[121] C. Poli, M. Bellec, U. Kuhl, F. Mortessagne, and $\mathrm{H}$. Schomerus, Selective enhancement of topologically induced interface states in a dielectric resonator chain, Nat. Commun. 6, 6710 (2015).

[122] P. St-Jean, V. Goblot, E. Galopin, A. Lemaitre, T. Ozawa, L. Le Gratiet, I. Sagnes, J. Bloch, and A. Amo, Lasing in topological edge states of a one-dimensional lattice, Nat. Photonics 11, 651 (2017).

[123] M. Parto, S. Wittek, H. Hodaei, G. Harari, M. A. Bandres, J. Ren, M. C. Rechtsman, M. Segev, D. N. Christodoulides, and M. Khajavikhan, Edge-Mode Lasing in 1D Topological Active Arrays, Phys. Rev. Lett. 120, 113901 (2018).

[124] H. Zhao, P. Miao, M. H. Teimourpour, S. Malzard, R. ElGanainy, H. Schomerus, and L. Feng, Topological hybrid silicon microlasers, Nat. Commun. 9, 981 (2018).

[125] B. Bahari, A. Ndao, F. Vallini, A. E. Amili, Y. Fainman, and B. Kanté, Nonreciprocal lasing in topological cavities of arbitrary geometries, Science 358, 636 (2017).

[126] G. Harari, M. A. Bandres, Y. Lumer, M. C. Rechtsman, Y. D. Chong, M. Khajavikhan, D. N. Christodoulides, and M. Segev, Topological insulator laser: Theory, Science 359, eaar4003 (2018); M. A. Bandres, S. Wittek, G. Harari, M. Parto, J. Ren, M. Segev, D. N. Christodoulides, and M. Khajavikhan, Topological insulator laser: Experiments, ibid. 359, eaar4005 (2018).

[127] Y. V. Kartashov and D. V. Skryabin, Two-Dimensional Topological Polariton Laser, Phys. Rev. Lett. 122, 083902 (2019).

[128] Y. Ota, K. Takata, T. Ozawa, A. Amo, Z. Jia, B. Kante, M. Notomi, Y. Arakawa, and S. Iwamoto, Active topological photonics, Nanophotonics 9, 547 (2020).

[129] F. Yang, Y.-C. Liu, and L. You, Anti- $\mathcal{P} \mathcal{T}$ symmetry in dissipatively coupled optical systems, Phys. Rev. A 96, 053845 (2017).

[130] V. V. Konotop and D. A. Zezyulin, Odd-Time Reversal $\mathcal{P} \mathcal{T}$ Symmetry Induced by an Anti- $\mathcal{P} \mathcal{T}$-Symmetric Medium, Phys. Rev. Lett. 120, 123902 (2018).

[131] Y. Li, Y.-G. Peng, L. Han, M.-A. Miri, W. Li, M. Xiao, X.-F. Zhu, J. Zhao, A. Alù, S. Fan, and C.-W. Qiu, Anti-parity-time symmetry in diffusive systems, Science 364, 170 (2019). 
[132] S. Ke, D. Zhao, J. Liu, Q. Liu, Q. Liao, B. Wang, and P. Lu, Topological bound modes in anti- $\mathcal{P} \mathcal{T}$-symmetric optical waveguide arrays, Opt. Express 27, 13858 (2019).

[133] H. Zhang, R. Huang, S.-D. Zhang, Y. Li, C.-W. Qiu, F. Nori, and H. Jing, Breaking Anti- $\mathcal{P} \mathcal{T}$ Symmetry by Spinning a Resonator, Nano Lett. 20, 7594 (2020).

[134] H. R. Jahromi, F. Nori, and R. L. Franco, Witnessing nonMarkovianity and criticality in (anti-)parity-time-symmetric systems, arXiv:2101.04663.
[135] Y. X. Zhao and A. P. Schnyder, Nonsymmorphic symmetryrequired band crossings in topological semimetals, Phsy. Rev. B 94, 195109 (2016).

[136] Y.-L. Zhang, R. P. H. Wu, A. Kumar, T. Si, and K. H. Fung, Nonsymmorphic symmetry-protected topological modes in plasmonic nanoribbon lattices, Phys. Rev. B 97, 144203 (2018).

[137] K. Takata and M. Notomi, Photonic Topological Insulating Phase Induced Solely by Gain and Loss, Phys. Rev. Lett. 121, 213902 (2018). 